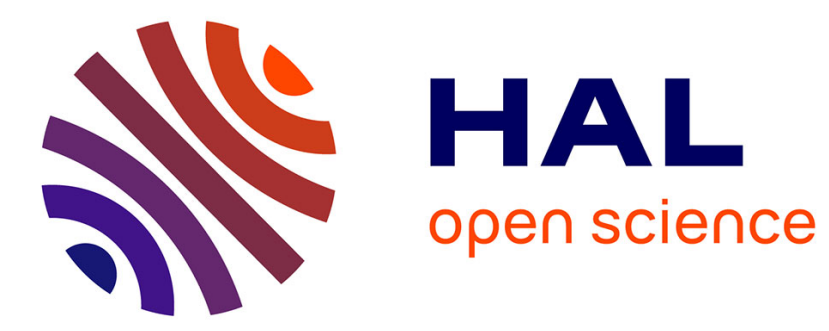

\title{
All-optical control of pattern dynamics generated by Airy beams
}

Lamyae Drouzi, Jordan Maufay, Marc Sciamanna, Delphine Wolfersberger, Nicolas Marsal

\section{- To cite this version:}

Lamyae Drouzi, Jordan Maufay, Marc Sciamanna, Delphine Wolfersberger, Nicolas Marsal. Alloptical control of pattern dynamics generated by Airy beams. Optics Letters, 2020, 45 (1), pp.9. 10.1364/OL.45.000009 . hal-02410549

\section{HAL Id: hal-02410549 \\ https://hal.science/hal-02410549}

Submitted on 1 Dec 2020

HAL is a multi-disciplinary open access archive for the deposit and dissemination of scientific research documents, whether they are published or not. The documents may come from teaching and research institutions in France or abroad, or from public or private research centers.
L'archive ouverte pluridisciplinaire HAL, est destinée au dépôt et à la diffusion de documents scientifiques de niveau recherche, publiés ou non, émanant des établissements d'enseignement et de recherche français ou étrangers, des laboratoires publics ou privés. 


\title{
All-optical control of pattern dynamics generated by Airy beams
}

\author{
Lamyae Drouzi, ${ }^{1,2, *}$ Jordan Maufay, ${ }^{1,2}$ Marc Sciamanna, ${ }^{1,2}$ Delphine Wolfersberger, ${ }^{1,2}$ and Nicolas Marsal ${ }^{1,2}$ \\ ${ }^{1}$ Chaire Photonique, LMOPS, CentraleSupélec, Université Paris Saclay, 2 rue Edouard Belin, 57070 Metz, France \\ ${ }^{2}$ Université de Lorraine, CentraleSupélec, LMOPS, 2 rue Edouard Belin, 57070 Metz, France
}

\begin{abstract}
We study a pattern forming system driven by two counter propagating 2D Airy beams in a nonlinear single feedback configuration. When increasing the Airy beam power, modulation instability takes place but with several successive instability thresholds that correspond to destabilization of the different Airy beam satellite lobes. Most importantly, the self-organization is accompanied by a natural drifting dynamics related to the intrinsic acceleration of the Airy beam. The drifting dynamics is controlled by the Airy beam parameters. Numerical simulations reproduce the experimental findings.
\end{abstract}

Controlling light by light is of peculiar interest in modern optics. Nonlinear optics allows for example generating spontaneous light self-organized structures (patterns) that can be controlled all-optically. Examples are shown in optical nonlinear cavity [1-3] as well as in single feedback configuration [4-9]. Instead of the conventionally used Gaussian beams, we suggested in [10] to use a beam carrying an orbital angular momentum (an optical vortex) to drive the pattern forming system and resulting in what we called a vortex induced rotation dynamics of optical patterns [10]. In what follows, we propose to use the peculiar properties of another unconventional beam, an Airy beam, to induce new interesting pattern dynamics.

The Airy wavepacket was discovered for the first time, in 1979, by Berry and Balazs [11] as a solution of the Schrodinger equation describing a free particle. As it possesses an infinite energy, it was impossible to generate it experimentally. We had to wait for almost three decades before its first realization in optics [12]. Although being a truncated solution of the ideal Airy wavepacket, the optical Airy beam keeps its non-spreading, self-healing and accelerating properties over a finite distance. An optical Airy beam is generated by linear and nonlinear methods $[13,14]$, and are performed with different apodization technics [15] to limit its energy. Scientists were then interested to control the Airy shape, its intensity and its acceleration [16-19]. Thanks to these characteristics, Airy beams have received growing interest due to their promising applications in all-optical routing [20,21], optical multiplexing [22], optical micromanipulation [23, 24], vacuum electron acceleration [25], plasmonic energy routing $[26,27]$ and soliton generation [28]. Very recently, the Airy beam has also been used in free space optical communication [29] .

In the present paper, we demonstrate that an Airy beam can self organize into a pattern with an intrinsic drifting dynamics. The generated pattern is naturally drifting and originates from a multi-thresholds bifurcation scenario related to the non-simultaneous destabilization of the different lobes of the Airy beam. Then, we highlight that the intrinsic Airy acceleration is correlated to the velocity of the drifting pattern and consequently that the transverse pattern velocity can be controlled via the longitudinal acceleration. we therefore demonstrate an all-optical control of optical pattern dynamics by tailoring the parameters of an optical Airy beam.

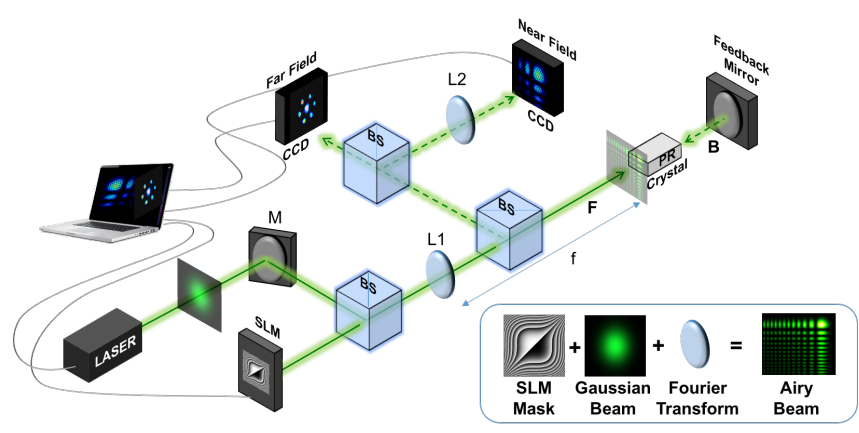

FIG. 1. Experimental set-up for pattern generation and measurements. BS, beam splitter; SLM, spatial light modulator; L1, L2, lens; M, mirror. The inset describes how the Airy beam is generated via the combination of a collimated Gaussian beam coming from the Laser, a peculiar phase profile launched onto the SLM and a Fourier transformation via the lens L1.

Our experiments (Fig. 1) are performed in a nonlinear Cobalt-doped Barium Titanate photorefractive crystal (BaTiO3:Co) with dimensions $6^{*} 6^{*} 6 \mathrm{~mm}^{3}$. We use a $532 \mathrm{~nm}$ coherent linearly polarized and collimated laser beam. We generate the truncated Airy beam by applying to a Gaussian laser beam a cubic phase pattern via a spatial light modulator (SLM). The Airy beam is then created in the Fourier plane of the lens $L_{1}$ where is located the input face of the crystal. A feedback mirror is placed at the crystal's back face. Due to the two-wave mixing process arising from the forward Airy beam $F$ and the backward Airy beam $B$ we expect that modulation instability takes place in the resulting transverse intensity profile of the two counter-propagating beams. The orientation of the crystal is chosen to provide a maximum energy coupling between the two counter-propagating beams. Consequently the angle between the optical axis of the system and the polarization $c$-axis of the crystal is set to approximately 25 degrees. The large electro-optic 
coefficient $r_{22}$ of the crystal gives then a strong contribution to the photorefractive coupling strength, providing a two-wave mixing amplification process in the backward direction $B$ [30]. In this study, the mirror is kept as straight as possible such as the forward $(F)$ and the backward $(B)$ beams are perfectly aligned. Observations and studies onto the near- and corresponding far-fields are realized by imaging the backward beam $B$, after its passage through the crystal, onto two CCD cameras (Fig. 1).

We first analyze the possibility to generate a pattern from a truncated Airy beam. We remind that the mirror is placed exactly at the back side of the crystal and it is perfectly aligned to guarantee an optimized overlap between $F$ and $B$. The main lobe size of the forward Airy beam is $x_{0}=95 \mu \mathrm{m}$ and the input power of the laser is increased gradually. Above a certain threshold $\left(P_{t h}=0.3\right.$ $\mathrm{mW})$, the wavevectors associated with the lowest gain exponentially grow following the principle of a winnertakes-all dynamics. As we can see in Fig. 2(a), the bifurcation from an homogeneous state [Fig. 2(a)(1)] to a modulated one [Fig. 2(a)(2)] is sub-critical. For increasing and decreasing values of the input power (ranging from $\mathrm{P}=0.3 \mathrm{~mW}$ to $\mathrm{P}=1.6 \mathrm{~mW}$ ), the bifurcation diagram presents an area where the pattern is unstable and coexists with the homogeneous state. Such a bistability has already been observed in classical pattern forming systems using Gaussian beams interaction [31, 32], but interestingly in our configuration the different lobes of the Airy beam do not destabilize simultaneously: the bifurcation curve has as many thresholds as visible lobes onto the camera [Fig. 2(a)(1)-(4)]. This bifurcation scenario is unique and originates from the peculiar intensity distribution of the Airy profile. The second, third and fourth lobes being smaller and smaller in size, need more and more gain (input power) to satisfy the modulational instability threshold.

Similarly to Gaussian beams in this configuration, the near- and far-field patterns present an honeycomb and respectively hexagonal structures [Fig. 2(a)(1)-(4), (b)]. As we also know from previous studies $[8,9]$, patterns are in principle static, in the single feedback configuration, unless transverse symmetry is broken e.g. by an unintentional misalignment of the feedback mirror. The forward and backward Airy beams are supposed to be perfectly aligned in our case. Still, the patterns produced by the counter-propagating beams interaction move naturally without any external forcing. Moreover, the drift follows the bisector direction corresponding exactly to the direction of the transverse Airy beam acceleration [Fig. 2 (b)]. We may conclude that the Airy beam controls its drifting pattern, but to be sure that it is not due to a misalignment in our experimental system, we complete our study by simulating the spatio-temporal dynamics of the pattern using the equations system (Eqs. 1). To fit also with the experimental curve [Fig. 2 (a)], we simulated the bifurcation scenario. Such a configu- ration can be modelled by the following three coupled equations [33]:

$$
\begin{aligned}
& \frac{\partial F}{\partial z}=-i D \frac{\partial^{2} F}{\partial x \partial y}+Q B \\
& \frac{\partial B}{\partial z}=-i D \frac{\partial^{2} B}{\partial x \partial y}+Q^{*} F \\
& \frac{\partial Q}{\partial t}=-Q+\gamma \frac{F B^{*}}{|F|^{2}+|B|^{2}+I_{d}}
\end{aligned}
$$

where $F$ and $B$ stand for the forward and the backward optical fields. D is the diffraction coefficient and $Q$ represents the complex amplitude of the photorefractive reflection grating arising inside the material. $z$ is the normalized propagation coordinate, $x$ and $y$ are the transverse ones and $\gamma$ is the photorefractive coupling strength. $I_{d}$ corresponds to the background illumination intensity which we neglect. The forward beam $F$ used in our simulation is defined by the initial conditions in $\mathrm{z}=0$, as :

$$
\left.F(x, y)=A i\left(\frac{x}{x_{0}}\right) A i\left(\frac{y}{y_{0}}\right) \exp \left(\frac{a x}{x_{0}}+\frac{a y}{y_{0}}\right)\right)
$$

where $a$ is a decay factor limiting the beam energy. $x_{0}$ and $y_{0}$ are the transverse scales in both $\mathrm{x}$ and $\mathrm{y}$ coordinates. We suppose then that $x_{0}=y_{0}$. Note that when we neglect the decay parameter $(a=0)$, we find the ideal Airy beam form. By using a conventional split step Fourier beam propagation method (BPM), we integrate the master equations (Eqs. 1) using the Airy beam $F$ (Eq. 2) and the backward beam $B$ linked to $F$ using a boundary condition as described in [33]:

$$
B(x, y, L, t)=-\sqrt{R} T_{F}^{-1} \exp \left(i \phi_{k}\right) T_{F}(F(x, y, L, t))
$$

Where $T_{F}$ is the transverse Fourier transform, L being the crystal length, $\mathrm{R}$ is the mirror reflectivity and $\phi_{k}$ denotes the propagation phase.

In the boundary conditions, we set the angle between the two counter-propagating Airy beams equal to 0 to simulate a perfectly aligned experimental condition. The simulations confirm that the instabilities drift towards the direction of the Airy beam transverse acceleration [white arrow in Fig. 2 (b)]. Thanks to equivalence principle, it has been demonstrated [34] that, in an accelerated frame, the Airy function presents an extra phase factor depending on the propagation variable z. Thus, the wavefront is tilted from the vertical direction $(\mathrm{z}=0)$. Hence, when we are placed on the output face of the crystal $(\mathrm{z}=0)$, we are observing an out of phase grating index of the equiphase direction, supposed to be the plane formation of the grating index. Hence, the intrinsic accelerating property of the Airy beam explains why the resulting pattern is moving. The numerical plot (Fig. 3) reproduces qualitatively well the experimental curve. It shows the normalized output intensity (intensity of the 

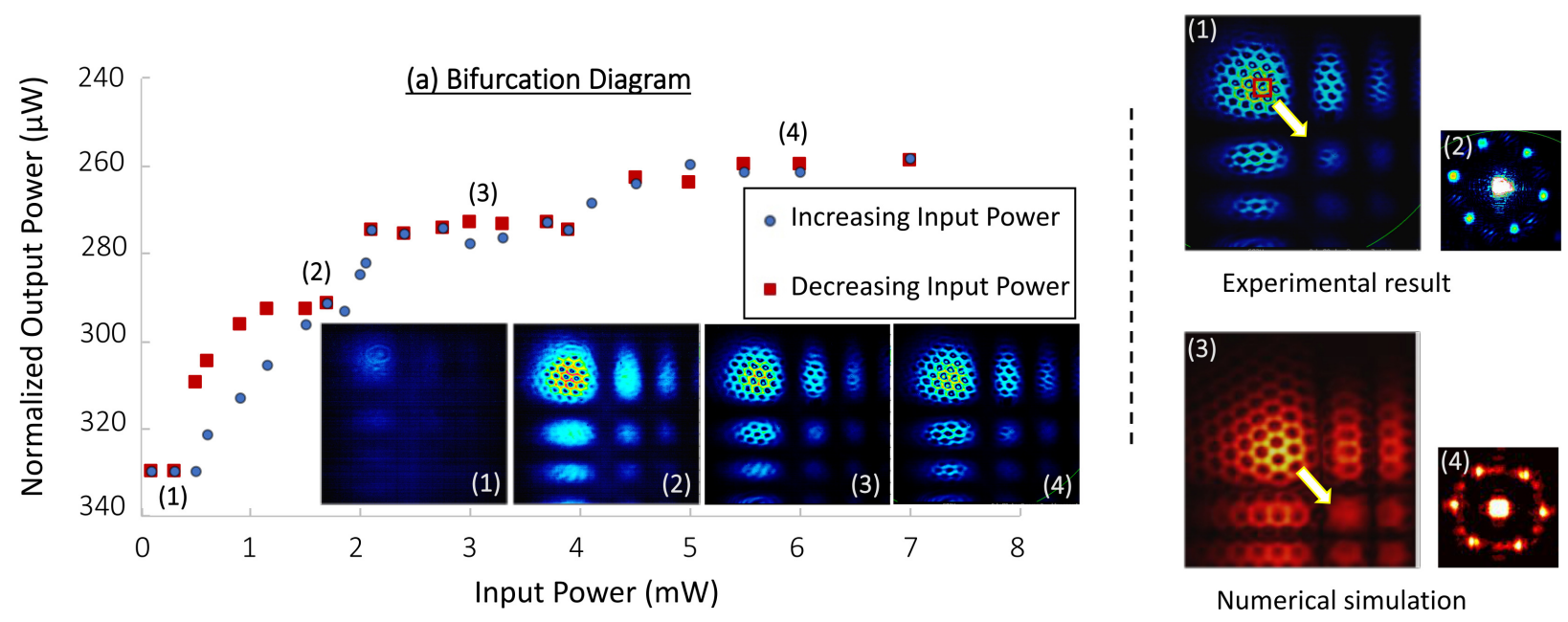

FIG. 2. (a) Experimental multi-thresholds bifurcation diagram of an Airy beam with $x_{0}=95 \mu \mathrm{m}$, and images in the near field obtained after each bifurcation. (b-1) Experimental and (b-3) numerical near-field patterns. (b-2) Experimental and (b-4) numerical far-field. Measurements are taken at the crystal back face. The white arrow illustrates the direction of the pattern drift.

overall unstable pattern in far-field divided by the intensity of the central spot corresponding to the stable forward Airy beam) versus the photorefractive coupling strength. We clearly identify the different bistability areas and jumps referring to the successive destabilization of the different Airy lobes.

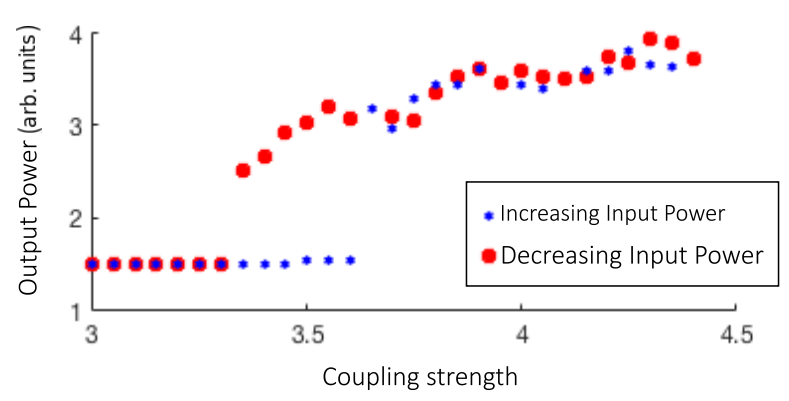

FIG. 3. Numerical multi-thresholds bifurcation diagram of a 2D Airy beam : Normalized pattern power at the crystal back face as a function of the photorefractive coupling strength $\gamma$.

Note that all the observations referring to the spatiotemporal dynamics are realized with the near-field intensity profiles. The corresponding far-fields are also accompanied by a dynamics but which is not clearly identifiable. In what follows we therefore decide to concentrate our study onto the dynamics of the near-field drifting instabilities for different Airy beam transverse accelerations. We want to identify the role played by the Airy acceleration on its pattern dynamics. Note that we can retrieve the Airy acceleration value $a_{c c}$ from its deflection $a_{0}$ using the following equation $a_{c c}=\frac{2 a_{0}}{z^{2}}$ [14]. Hence, for a fixed z, deflection and acceleration are proportional. We have summarized in figure 4 the pattern drift velocity versus four different Airy deflections $a_{0}$. Experimentally and numerically changing the transverse Airy deflection consists of varying the width of the Airy main lobe $x_{0}$ as they are linked by the relation $a_{0}=\frac{\sqrt{2} z^{2}}{4 k^{2} x_{0}^{3}}[14]$. By knowing the distance traveled by one instability spot [red squares in Fig. 2(b)] and the time separation between two consecutive near-field pictures, one can calculate the velocity of the drift. We have normalized the size of the main lobe and kept the power of the input Airy beam constant. Deflections are normalized as well. Note that the deflection's order of magnitude in our experiments is around nanometers. We observe experimentally [Fig. 4 (a)] that the velocity of the drifting pattern grows as the deflection increases. For instance, for $a_{0}=0.58$, the pattern moves with $v=6.87 \mu \mathrm{m} . \mathrm{s}^{-1}$ whereas for $a_{0}=1.57$ the pattern velocity is equal to $v=10.49$ $\mu \mathrm{m} . s^{-1}$. To ensure that the increase of velocity is essentially due to the Airy acceleration process, we performed numerical simulation and made the same analysis onto the corresponding near-field profiles for the same values of the photorefractive coupling strength $\gamma$. The numerical results [Fig. 4 (b)] are in excellent agreement with experiment [Fig. 4 (a)]. Increasing the deflection corresponds implicitly to saying that we increase the Airy phase shift [34]. Therefore, explaining why the pattern drifting velocity increases as well.

To summarize, we have explored both experimentally and numerically the formation and the control of optical pattern originating from an Airy beam in a single feedback configuration. We find that modulation instability 


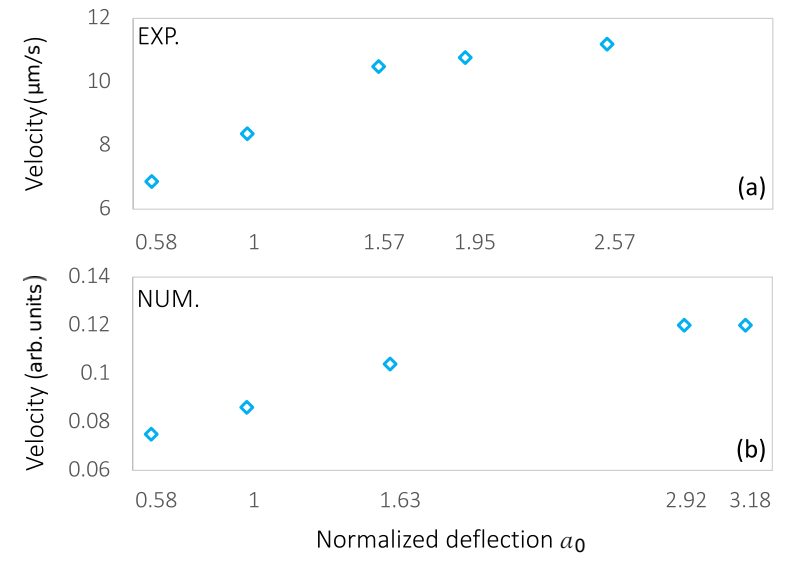

FIG. 4. (a) Experimental and (b) numerical velocity of the main lobe's Airy pattern as a function of the normalized Airy deflection $a_{0}$ at the crystal back face .

starts first in the main lobe of the Airy beam when we reach a certain value of the input power, while the others lobes still maintain their steady homogeneous state. By increasing the input power even more, we notice that the other lobes begin to destabilize, giving rise to other bifurcation thresholds. The resulting bifurcation curve, with a multi-thresholds shape, makes a signature of the Airy beam pattern formation. Furthermore, contrary to the pattern formation induced by a Gaussian beam, the Airy pattern presents a drifting dynamics in a preferential direction in absence of any external breaking of symmetry. Thus, the Airy beam translates its longitudinal acceleration into a transverse drift by the means of a phase shift. We have measured that the transverse pattern velocity is proportional to the Airy acceleration thanks to the correlation between its longitudinal phase shift and its transverse deflection. This work therefore demonstrates an all-optical control of the dynamics of optical patterns by tailoring the parameters of an accelerating beam.

The authors acknowledge the support of Fondation Supélec, AIRBUS-GDI Simulation, Metz Métropole, Conseil Départemental de Moselle, Conseil Régional Grand-Est, Préfecture de Région Grand-Est and FEDER through the funding of the Chair in Photonics. The authors also ackowledge Thomas Bouchet for fruitful discussions.

* lamyae.drouzi@centralesupelec.fr

[1] Mark Saffman, Don Montgomery, and Dana Z Anderson. Collapse of a transverse-mode continuum in a selfimaging photorefractively pumped ring resonator. Optics letters, 19(8):518-520, 1994.

[2] R Kuszelewicz, I Ganne, I Sagnes, G Slekys, and M Brambilla. Optical self-organization in bulk and multiquantum well GaAlAs microresonators. Physical review letters, 84(26):6006, 2000.

[3] Umberto Bortolozzo, A Montina, Fortunato Tito Arecchi, JP Huignard, and Stefania Residori. Spatiotemporal pulses in a liquid crystal optical oscillator. Physical review letters, 99(2):023901, 2007.

[4] G d'Alessandro and WJ Firth. Spontaneous hexagon formation in a nonlinear optical medium with feedback mirror. Physical review letters, 66(20):2597, 1991.

[5] G d'Alessandro and WJ Firth. Hexagonal spatial patterns for a kerr slice with a feedback mirror. Physical Review A, 46(1):537, 1992.

[6] Eric Louvergneaux. Pattern-dislocation-type dynamical instability in 1d optical feedback kerr media with gaussian transverse pumping. Physical review letters, 87(24):244501, 2001.

[7] Stefania Residori. Patterns, fronts and structures in a liquid-crystal-light-valve with optical feedback. Physics Reports, 416(5-6):201-272, 2005.

[8] Nicolas Marsal, Delphine Wolfersberger, Marc Sciamanna, Germano Montemezzani, and DN Neshev. Experimental control of pattern formation by photonic lattices. Optics letters, 33(21):2509-2511, 2008.

[9] Nicolas Marsal, Delphine Wolfersberger, Marc Sciamanna, and Germano Montemezzani. Noise-and dynamics-sustained patterns in a nonlinear photorefractive system. Physical Review A, 81(3):031804, 2010.

[10] Vianney Caullet, Nicolas Marsal, Delphine Wolfersberger, and Marc Sciamanna. Vortex induced rotation dynamics of optical patterns. Physical review letters, 108(26):263903, 2012.

[11] Michael V Berry and Nandor L Balazs. Nonspreading wave packets. American Journal of Physics, 47(3):264267, 1979.

[12] GA Siviloglou, J Broky, Aristide Dogariu, and DN Christodoulides. Observation of accelerating airy beams. Physical Review Letters, 99(21):213901, 2007.

[13] Tal Ellenbogen, Noa Voloch-Bloch, Ayelet GananyPadowicz, and Ady Arie. Nonlinear generation and manipulation of airy beams. Nature photonics, 3(7):395, 2009.

[14] Dong Wei, Ya Yu, Mingtao Cao, Liyun Zhang, Fengjuan Ye, Wenge Guo, Shougang Zhang, Hong Gao, and Fuli Li. Generation of airy beams by four-wave mixing in rubidium vapor cell. Optics letters, 39(15):4557-4560, 2014.

[15] Michel Zamboni-Rached, Kleber Zuza Nóbrega, and César Augusto Dartora. Analytic description of airytype beams when truncated by finite apertures. Optics Express, 20(18):19972-19977, 2012.

[16] Yi Hu, Simon Huang, Peng Zhang, Cibo Lou, Jingjun $\mathrm{Xu}$, and Zhigang Chen. Persistence and breakdown of airy beams driven by an initial nonlinearity. Optics letters, 35(23):3952-3954, 2010.

[17] Nikolaos K Efremidis. Airy trajectory engineering in dynamic linear index potentials. Optics letters, 36(15):3006-3008, 2011.

[18] Zhuoyi Ye, Sheng Liu, Cibo Lou, Peng Zhang, Yi Hu, Daohong Song, Jianlin Zhao, and Zhigang Chen. Acceleration control of airy beams with optically induced refractive-index gradient. Optics letters, 36(16):32303232, 2011.

[19] Sabino Chávez-Cerda, Ulises Ruiz, Victor Arrizón, and Héctor M Moya-Cessa. Generation of airy solitary-like wave beams by acceleration control in inhomogeneous 
media. Optics express, 19(17):16448-16454, 2011.

[20] Patrick Rose, Falko Diebel, Martin Boguslawski, and Cornelia Denz. Airy beam induced optical routing. Applied Physics Letters, 102(10):101101, 2013.

[21] Noémi Wiersma, Nicolas Marsal, Marc Sciamanna, and Delphine Wolfersberger. All-optical interconnects using airy beams. Optics letters, 39(20):5997-6000, 2014.

[22] Thomas Bouchet, Nicolas Marsal, Marc Sciamanna, and Delphine Wolfersberger. Light-induced interconnects using nonlinear airy beam interactions. Journal of Physics: Photonics, 2018.

[23] Peng Zhang, Jai Prakash, Ze Zhang, Matthew S Mills, Nikolaos K Efremidis, Demetrios N Christodoulides, and Zhigang Chen. Trapping and guiding microparticles with morphing autofocusing airy beams. Optics letters, 36(15):2883-2885, 2011.

[24] Zhu Zheng, Bai-Fu Zhang, Hao Chen, Jianping Ding, and Hui-Tian Wang. Optical trapping with focused airy beams. Applied optics, 50(1):43-49, 2011.

[25] Jian-Xing Li, Xiao-Long Fan, Wei-Ping Zang, and JianGuo Tian. Vacuum electron acceleration driven by two crossed airy beams. Optics letters, 36(5):648-650, 2011.

[26] Alessandro Salandrino and Demetrios N Christodoulides. Airy plasmon: a nondiffracting surface wave. Optics letters, 35(12):2082-2084, 2010.

[27] Alexander Minovich, Angela E Klein, Norik Janunts, Thomas Pertsch, Dragomir N Neshev, and Yuri S Kivshar. Generation and near-field imaging of airy sur- face plasmons. Physical review letters, 107(11):116802, 2011.

[28] Ido Kaminer, Mordechai Segev, and Demetrios N Christodoulides. Self-accelerating self-trapped optical beams. Physical review letters, 106(21):213903, 2011.

[29] Guoxuan Zhu, Yuanhui Wen, Xiong Wu, Yujie Chen, Jie Liu, and Siyuan Yu. Obstacle evasion in free-space optical communications utilizing airy beams. Optics letters, 43(6):1203-1206, 2018.

[30] Germano Montemezzani. Optimization of photorefractive two-wave mixing by accounting for material anisotropies: Knbo 3 and batio 3. Physical Review A, 62(5):053803, 2000.

[31] Serguey G Odoulov, Mikhail Yu Goulkov, and Oksana A Shinkarenko. Threshold behavior in formation of optical hexagons and first order optical phase transition. Physical review letters, 83(18):3637, 1999.

[32] Nicolas Marsal, Lionel Weicker, Delphine Wolfersberger, and Marc Sciamanna. Bistability controlled by convection in a pattern-forming system. Physical review letters, 118(1):013902, 2017.

[33] O Sandfuchs, F Kaiser, and MR Belić. Self-organization and fourier selection of optical patterns in a nonlinear photorefractive feedback system. Physical Review A, 64(6):063809, 2001.

[34] Daniel M Greenberger. Comment onnonspreading wave packets. American Journal of Physics, 48(3):256-256, 1980. 